\title{
Oxide Heterostructures for Efficient Solar Cells
}

\author{
Elias Assmann, ${ }^{1}$ Peter Blaha, ${ }^{2}$ Robert Laskowski, ${ }^{2}$ Karsten Held, ${ }^{1}$ Satoshi Okamoto $,{ }^{3}, *$ and Giorgio Sangiovanni ${ }^{4}$ \\ ${ }^{1}$ Institute of Solid State Physics, Vienna University of Technology, 1040 Vienna, Austria \\ ${ }^{2}$ Institute of Materials Chemistry, Vienna University of Technology, 1040 Vienna, Austria \\ ${ }^{3}$ Materials Science and Technology Division, Oak Ridge National Laboratory, Oak Ridge, Tennessee 37831, USA \\ ${ }^{4}$ Institut für Theoretische Physik und Astrophysik, Universität Würzburg, 97074 Würzburg, Germany
}

(Dated: April 9, 2013)

\begin{abstract}
We propose an unexplored class of absorbing materials for high-efficiency solar cells: heterostructures of transition-metal oxides. In particular, $\mathrm{LaVO}_{3}$ grown on $\mathrm{SrTiO}_{3}$ has a direct band gap $\sim 1.1 \mathrm{eV}$ in the optimal range as well as an internal potential gradient, which can greatly help to separate the photogenerated electron-hole pairs. Furthermore, oxide heterostructures afford the flexibility to combine $\mathrm{LaVO}_{3}$ with other materials such as $\mathrm{LaFeO}_{3}$ in order to achieve even higher efficiencies with band-gap graded solar cells. We use density-functional theory to demonstrate these features.
\end{abstract}

PACS numbers: 88.40.fh, 73.20.At, 78.20.Bh

The stunning discovery by Ohtomo and Hwang $[1,2]$ that the interface between the band insulators $\mathrm{SrTiO}_{3}$ and $\mathrm{LaAlO}_{3}$ (or $\mathrm{LaTiO}_{3}$ ) can become conductive above a critical thickness of the latter opened the research field of layered oxide heterostructures. The interest comes not only from novel physical effects that are absent in the constituent bulk materials, but also from the perspective of designing and tuning specific properties to achieve desired functionalities. This flexibility becomes even more pronounced when materials with partially filled $d$-shells, and hence strong electronic correlations, are involved $[3,4]$. In particular, these novel oxide heterostructures raise hope to surpass state-of-the-art semiconductors for specific applications. In this Letter, we propose highefficiency solar cells as such an application.

The performance of photovoltaic systems is characterized by the efficiency $\eta$ of conversion of the incident photon energy to electrical power. A natural limit for $\eta$ is set by the optical gap $\Delta$ of the absorbing material: photons with energy $\hbar \omega<\Delta$ are not absorbed at all; those with $\hbar \omega>\Delta$ may be absorbed but each only contributes an amount of energy equal to $\Delta$, with the difference being lost to relaxation processes, i.e., heat generation. This consideration, combined with further loss channels, leads to the famous Shockley-Queisser limit [5], which gives an upper bound for $\eta$ as a function of $\Delta$ with $\eta \lesssim 34 \%$ in the optimum range $\Delta=1-1.5 \mathrm{eV}$. In the case of semiconductors, a great effort now concentrates on the reduction of so-called Shockley-Read-Hall electron-hole recombination caused by defect-induced "trap" states and, in general, any intrinsic recombination mechanism. Even small improvements on the final efficiency of a solar cell have an immense economical and environmental impact, and are intensively pursued by industry $[6,7]$.

Why oxide heterostructures? There are four properties that make layered oxide heterostructures, and in particular $\mathrm{LaVO}_{3} \mid \mathrm{SrTiO}_{3}$, a promising candidate for efficient solar cells (cf. Fig. 1): (i) an intrinsic electric field

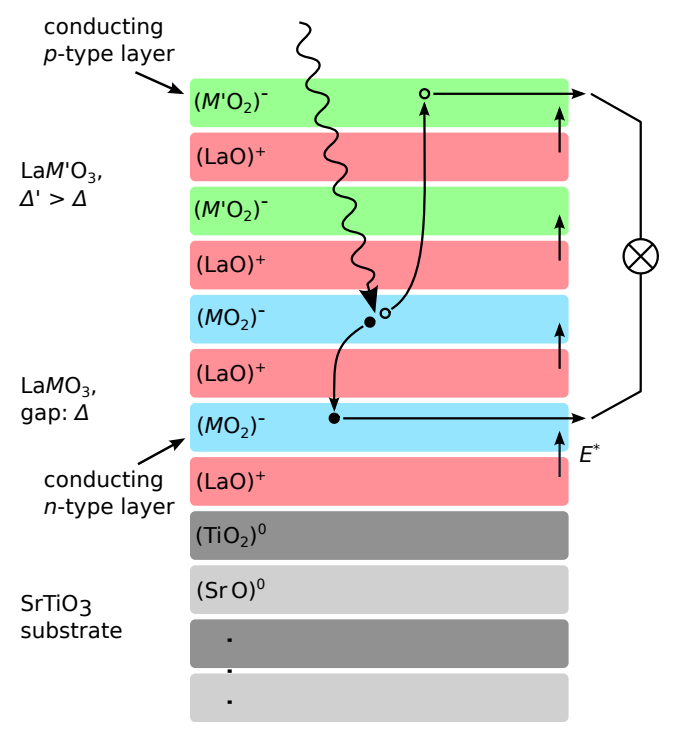

Figure 1: Schematic of a gap-graded oxide-heterostructure solar cell. The cell shown here combines two absorber materials, generically $\mathrm{La}^{\prime} \mathrm{O}_{3}$ and $\mathrm{La} \mathrm{O}_{3}\left(M\right.$ and $M^{\prime}$ being different transition metals); the compound with the larger band gap $\Delta^{\prime}>\Delta$ should face the sun. Incoming photons with energy $\hbar \omega>\Delta^{\prime}$ can be absorbed in the top material and contribute an energy $\Delta^{\prime}$, whereas those with $\Delta<\hbar \omega<\Delta^{\prime}$ traverse the first part, which is transparent to them, and can be absorbed in the lower material, contributing an energy $\Delta$. Once an electron-hole pair is dissociated, the built-in effective electric field $E^{*}$ will drive the carriers to their respective contacts, which are naturally provided in the form of conducting surface and interface layers.

emerges in the photoabsorbing region, which may efficiently separate photoexcited electrons and holes; (ii) the band gap of $1.1 \mathrm{eV}$ is direct and in the ideal energy range for harvesting the sunlight reaching the Earth's surface; (iii) interfaces and surfaces can become metallic on a thickness of about one unit cell, and those layers naturally allow for extracting the charge carriers; and (iv) one 


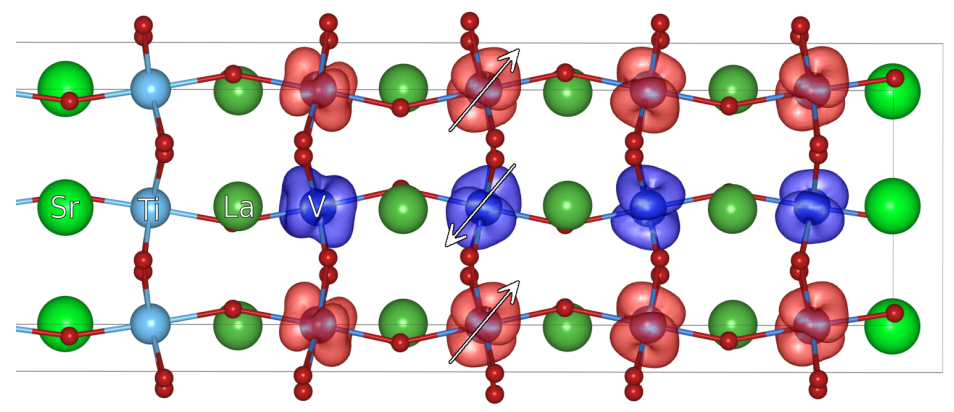

Figure 2: Multi-layer $\mathrm{LaVO}_{3} \mid \mathrm{SrTiO}_{3}$ heterostructure with $4 \mathrm{VO}_{2}$ planes. Superimposed on the relaxed structure, the red/blue (light/dark gray) lobes show isosurfaces of the positive/negative part of the spin-density $n_{\uparrow}-n_{\downarrow}$. Owing to the periodic repetition of the cell, both an $n$-type (left) and a $p$-type (right) interface appear. The substrate is only partly shown; altogether there are six Ti layers. As in bulk $\mathrm{LaVO}_{3}$, the V-d electrons show AF-C spin order; for the central V layers, the AF-G orbital order is similarly preserved.

can flexibly combine different materials [8-12] and grow heterostructures with multiple band gaps, e.g., alternate $\mathrm{LaVO}_{3}$ with $\mathrm{LaFeO}_{3}$ in order to realize a so-called bandgap graded design [13]. By means of density-functional theory (DFT) calculations we establish these properties of $\mathrm{LaVO}_{3} \mid \mathrm{SrTiO}_{3}$ and, given the enormous flexibility offered by oxide heterostructures, we suggest them as a new candidate for efficient photovoltaic devices.

$\mathrm{LaAlO}_{3} \mid \mathrm{SrTiO}_{3}$ can be considered the prototype for the kind of heterostructure we consider here, and has attracted by far the most attention [14-18]. However, for our purposes $\mathrm{LaVO}_{3} \mid \mathrm{SrTiO}_{3}$ is more suitable since $\mathrm{LaAlO}_{3} \mid \mathrm{SrTiO}_{3}$ has a band gap of several electron volts, much too large for photovoltaics. Both heterostructures share a feature which is most interesting in relation to the electron-hole recombination problem: a polar interface with an intrinsic electric field. In $\mathrm{LaVO}_{3}$ both $\mathrm{La}$ and $\mathrm{V}$ have nominal valence +3 such that the $\mathrm{LaO}$ planes are positively charged and the $\mathrm{VO}_{2}$ planes are negatively charged, inducing an electric field between these layers (depicted schematically in Fig. 1; for quantitative results see Fig. 4 below). In the substrate $\mathrm{SrTiO}_{3}$, on the other hand, Sr has nominal valence +2 and $\mathrm{Ti}$ has +4 , such that the $\mathrm{SrO}$ and $\mathrm{TiO}_{2}$ planes are both charge neutral. Based on these simple considerations one expects a potential gradient induced by the polar discontinuity between $\mathrm{LaVO}_{3}\left(\right.$ or $\mathrm{LaAlO}_{3}$ ) and $\mathrm{SrTiO}_{3}$ [19]. DFT calculations for $\mathrm{LaAlO}_{3} \mid \mathrm{SrTiO}_{3}$ show that, in order to avoid this so-called polarization catastrophe, the polar discontinuity is partially compensated by electrons transferred from the top of the heterostructure to the interface, whereas a substantial gradient persists. Recently, a finite potential gradient has also been measured experimentally [20]. That said, such measurements are challenging, in particular because of the difficulty of controlling oxygen vacancies in $\mathrm{LaAlO}_{3}$ (or, equally, $\mathrm{LaVO}_{3}$ ), which may greatly influence the gradient.

The built-in electric field will support the separation of photogenerated electron-hole pairs and will drive the dissociated charge carriers selectively to the respective contacts for extraction. $\mathrm{LaVO}_{3} \mid \mathrm{SrTiO}_{3}$ combines this feature with a near-optimum direct band gap of $1.1 \mathrm{eV}$. Therefore, we concentrate on this structure in the present paper.

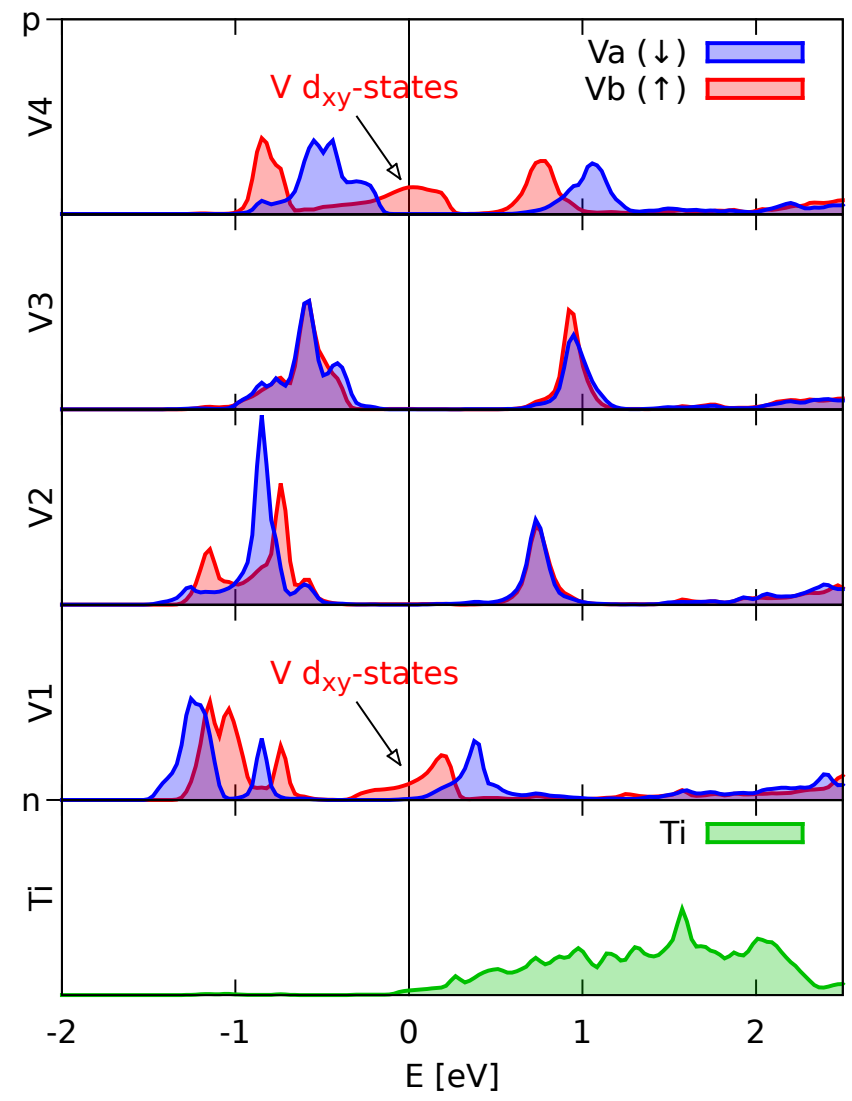

Figure 3: Density of states of a multi-layer $\mathrm{LaVO}_{3} \mid \mathrm{SrTiO}_{3}$ heterostructure. Each of the four $\mathrm{VO}_{2}$ layers (V1-V4) contains two inequivalent $\mathrm{V}$ atoms ( $\mathrm{Va}$ and $\mathrm{Vb}$ ) carrying opposite spin; the majority-spin $\mathrm{V}$ contribution is shown for each atom. For the Ti contribution (we show only the interfacial layer), the two spins as well as the two sites are essentially identical. These are the only relevant contributions around the Fermi level. Note the layer-by-layer shift of the bands which indicates the potential gradient, and the appearance of conducting states (of $d_{x y}$ character, only in one spin channel) on the interfacial $\mathrm{V}$.

Another advantage of the proposed oxide heterostructures for solar-cell applications is that electrical contacts to collect the photogenerated charge carriers are naturally provided. The (" $n$-type"; see below) $\mathrm{LaVO}_{3} \mid \mathrm{SrTiO}_{3}$ interface itself is metallic; in fact, it would be difficult to 


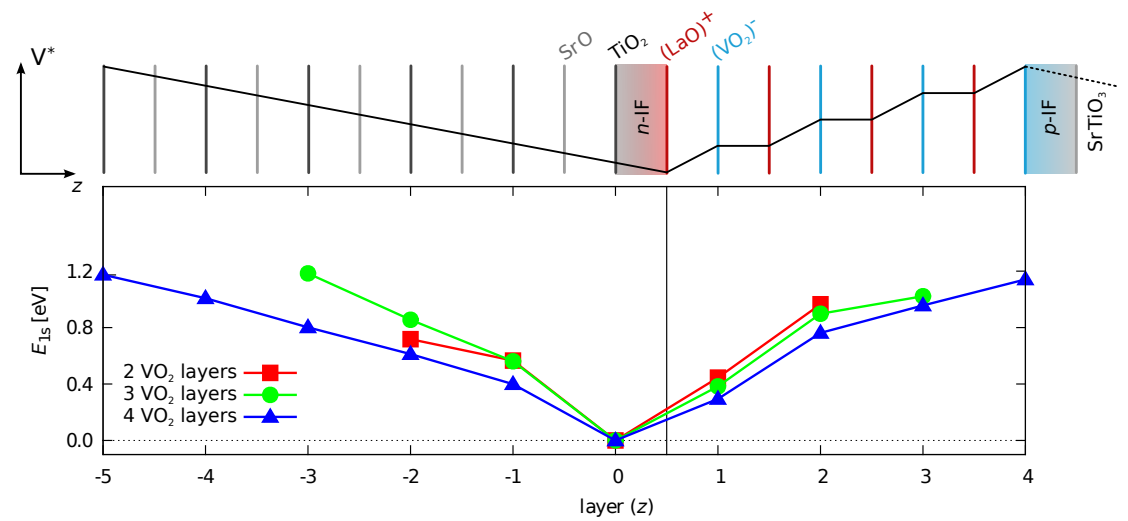

Figure 4: Potential gradient in multilayer $\mathrm{LaVO}_{3} \mid \mathrm{SrTiO}_{3}$ heterostructures. The curves $(\boldsymbol{\square} / \bullet / \Delta$ distinguish structure size) track the energy of an $\mathrm{O}-1 \mathrm{~s}$ state through the $\mathrm{TiO}_{2}$ (layer $\left.\leq 0\right)$ and $\mathrm{VO}_{2}$ (layer $>0$ ) layers, providing a measure of the potential gradient. Above the data, the layers, interfaces (IF), and effective electron potential $V^{*}$ are shown schematically. Periodicity forces the potential to return to zero in the $\mathrm{SrTiO}_{3}$ part after it has ramped up in the $\mathrm{LaVO}_{3}$ part. contact it otherwise. Our DFT calculations show that the surface layer is also metallic and, hence, suitable to extract the holes. However, surface defects and edges might localize the holes. Hence, careful surface preparation or even an additional metallic layer such as $\mathrm{SrVO}_{3}$ or other metallic contacts will be necessary on the surface side.

Ab initio simulations of oxide solar cells. We study structures with different numbers of $\mathrm{LaO}$ and $\mathrm{VO}_{2}$ layers grown on the (001) surface of $\mathrm{SrTiO}_{3}$ considering both $(i)$ periodically repeated arrangements containing one " $n$-type" $\left(\mathrm{TiO}_{2} \mid[\mathrm{LaO}]^{+}\right)$and one " $p$-type" $\left(\mathrm{SrO} \mid\left[\mathrm{VO}_{2}\right]^{-}\right)$interface and $(i i)$ an $n$-type $\left(\mathrm{TiO}_{2} \mid \mathrm{LaO}\right)$ interface with a slab of vacuum on top of the $\mathrm{LaVO}_{3}$ part to break periodicity. These setups are termed $(i)$ "multilayer" and (ii) "thin-film" geometries.

The DFT $+\mathrm{U}$ calculations were performed using the fullpotential linearized augmented plane-wave code WIEN2k [21], with the PBEsol [22] and modified Becke-Johnson [31] exchange-correlation potentials and a local Coulomb interaction term $U$ on $\mathrm{Ti}, \mathrm{V}$ and Fe. The absorption coefficients in Fig. 5 were calculated using the optic code for WiEn2k [23]. See Supplemental Material [24] for technical details, including a discussion of excitonic effects.

We begin by showing one representative $\mathrm{LaVO}_{3} \mid \mathrm{SrTiO}_{3}$ structure, including the results from structural optimization and electronic self-consistency, in Fig. 2. As in the bulk $[25,26]$, the V-d electrons show staggered spin and orbital order. The spin order is of the C-type (ferromagnetically coupled antiferromagnetic planes), while the orbital order is of the G-type (alternating in all directions) [27]. The $\mathrm{V}$ and Ti contributions to the density of states for this case are shown in Fig. 3, in the energy range of the $\mathrm{V}-d$ bands. These bands shift layer by layer, a first sign of the potential gradient. The gradient can be evidenced more clearly by tracking the energy of a core level throughout all layers, as shown in Fig. 4.

We estimate the potential slope in the $\mathrm{LaVO}_{3}$ region to be $0.08 \mathrm{eV} / \AA$ (see Fig. 4). A similar potential slope is observed in the $\mathrm{LaVO}_{3}$ region of thin-film structures (see Supplemental Fig. 1 [24]). As the photocarrier excitation takes place in the $\mathrm{LaVO}_{3}$ region, such a potential gradient inside $\mathrm{LaVO}_{3}$ regions helps to efficiently separate photoexcited electrons and holes.

For the multi-layer heterostructure with four $\mathrm{V}$ layers, we observe the appearance of states at the Fermi level at the interfaces between $\mathrm{LaVO}_{3}$ and $\mathrm{SrTiO}_{3}$. This confirms the scenario of metallic interfaces due to the electronic reconstruction found also in $\mathrm{LaAlO}_{3} \mid \mathrm{SrTiO}_{3}$ within DFT. Yet, contrary to $\mathrm{LaAlO}_{3} \mid \mathrm{SrTiO}_{3}$, the carriers reside mostly on the $\mathrm{V}$ rather than the $\mathrm{Ti}$, in accordance with recent experimental results [28]. The critical thickness that we find for multi-layer $\mathrm{LaVO}_{3} \mid \mathrm{SrTiO}_{3}$ heterostructures at the $\mathrm{DFT}+\mathrm{U}$ level is four. This result compares well with what has been reported in one experiment [29] and it is smaller than what was found in another experiment [28]. While any solar cell must have a band gap in order to generate electrical energy, conducting states that stay confined to the interface may in fact prove useful in extracting the photogenerated charge carriers.

Let us now turn to a central quantity for solar cell applications, the optical absorption (i.e. $\alpha$ in the BeerLambert law $I(r) \sim \mathrm{e}^{-\alpha r}$, see Supplemental Material for details [24]), shown in Fig. 5. A major advantage of $\mathrm{LaVO}_{3} \mid \mathrm{SrTiO}_{3}$ heterostructures over the current standard absorber material is that, contrary to $\mathrm{Si}$, the band gap in $\mathrm{LaVO}_{3}$ is direct, such that photons carrying the band-gap energy can create electron-hole pairs without the aid of phonons or another indirect scattering processes. Furthermore, across most of the solar spectrum, bulk $\mathrm{LaVO}_{3}$ compares favorably with $\mathrm{CdTe}$, a direct-gap material currently used for high-efficiency, thin-film solar cells.

The validity of the absorption coefficient computed within DFT is confirmed by a comparison to experimental data on bulk $\mathrm{LaVO}_{3}$ from Ref. 26. Note that the first sharp peak in the theoretical curve is very sensitive to details of the crystal structure (in the limit of a cubic unit cell, it vanishes by symmetry). This might explain why it is absent in the experiment and in the heterostructure calculation.

$\mathrm{LaVO}_{3} \mid \mathrm{SrTiO}_{3}$ heterostructures have similar optical properties as bulk $\mathrm{LaVO}_{3}$, with the additional freedom 


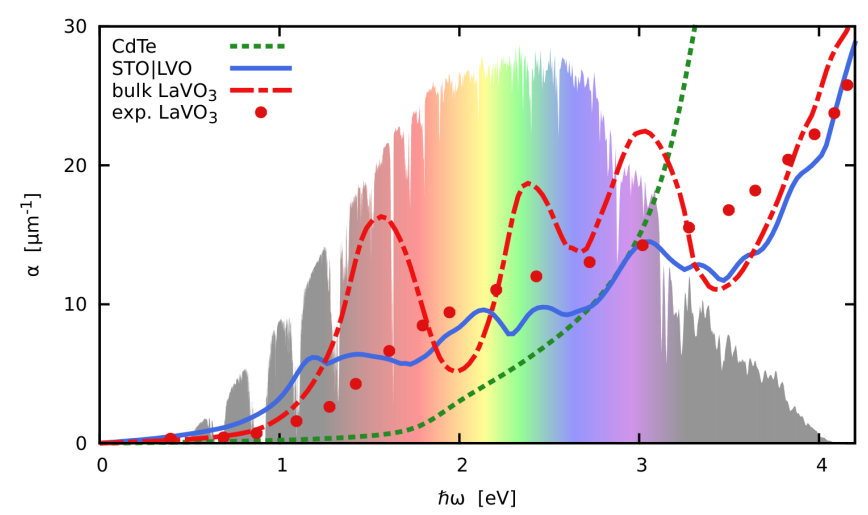

Figure 5: Absorption coefficients and solar spectrum. The lines show the absorption coefficients of bulk $\mathrm{LaVO}_{3}$ and $\mathrm{LaVO}_{3} \mid \mathrm{SrTiO}_{3}$ (for a multi-layer structure with $2 \mathrm{~V}$ layers) compared to experimental data on bulk $\mathrm{LaVO}_{3}[26]$ and a calculation for $\mathrm{CdTe}$, which is widely used in current highefficiency solar cells. In the background, the solar spectrum as measured on the Earth's surface is shown (standard global air mass 1.5 , in arbitrary units).

that individual layers can have different band gaps owing to layer-dependent distortion and resulting crystal-field splittings. Another advantage of oxide heterostructures is that we can flexibly combine $\mathrm{LaVO}_{3}$ with a second transition metal oxide with a somewhat larger band gap, such as $\mathrm{LaFeO}_{3}$, improving the conversion efficiency in the high-energy region of the solar spectrum. Thus one can construct gap-graded structures, in which the layers facing the sun have larger gaps than the layers underneath. The $\mathrm{SrTiO}_{3}$ substrate can be on either side because it is transparent to visible light. This scheme efficiently reduces the losses due to the magnitude of the band gap and provides the primary means to exceed the Shockley-Queisser limit. We perform DFT+U calculations for such a $\mathrm{LaFeO}_{3}\left|\mathrm{LaVO}_{3}\right| \mathrm{SrTiO}_{3}$ heterostructure and find a layer-dependent gap of $2.2 \mathrm{eV}$ for the $\mathrm{LaFeO}_{3}$ and $1.1 \mathrm{eV}$ for the $\mathrm{LaVO}_{3}$ part, in close agreement with the bulk values. See Supplemental Fig. 3 for details [24].

Note added in proof. Manousakis [Phys. Rev. B 82, 125109 (2010)] reports that, in a Mott insulator, a single photon may create multiple electron-hole pairs by impact ionization. This effect could further increase the efficiency of the $\mathrm{LaVO}_{3} \mid \mathrm{SrTiO}_{3}$ solar cell proposed here.

Acknowledgments. We thank Ralph Claessen, HoNyung Lee, Margherita Marsili, David Parker, Andrei Pimenov, Jens Pflaum and Zhicheng Zhong for fruitful discussions, and we acknowledge financial support from a Vienna University of Technology innovative project grant (E.A. and G.S.), SFB ViCoM [FWF project ID F4103N13] (P. B. and K. H.), and the Laboratory Directed Research and Development Program of ORNL (S.O.).
* okapon@ornl.gov

[1] A. Ohtomo, D. A. Muller, J. L. Grazul and H. Y. Hwang, Nature 419, 378 (2002).

[2] A. Ohtomo and H. Y. Hwang, Nature 427, 423 (2004).

[3] S. Okamoto and A. J. Millis, Nature 428, 630 (2004).

[4] A. J. Millis and D. G. Schlom, Phys. Rev. B 82, 073101 (2010)

[5] W. Shockley and H. J. Queisser, J. Appl. Phys. 32, 510 (1961).

[6] A. Polman and H. A. Atwater, Nature Mater. 11, 174 (2012).

[7] EPIA report, "Global Market Outlook for Photovoltaics until 2016". Downloadable from http://www.epia.org

[8] T. Koida, M. Lippmaa, T. Fukumura, K. Itaka, Y. Matsumoto, M. Kawasaki and H. Koinuma, Phys. Rev. B 66, 144418 (2002).

[9] I. Bozovic, G. Logvenov, M. A. J. Verhoeven, P. Caputo, E. Goldobin and T. H. Geballe, Nature 422, 873 (2003).

[10] Z. Sefrioui, D. Arias, V. Peña, J. E. Villegas, M. Varela, P. Prieto, C. Leon, J. L. Martinez and J. Santamaria, Phys. Rev. B 67, 214511 (2003).

[11] P. Yu, J.-S. Lee, S. Okamoto, M. D. Rossell, M. Huijben, C.-H. Yang, Q. He, J. X. Zhang, S. Y. Yang, M. J. Lee, Q. M. Ramasse, R. Erni, Y.-H. Chu, D. A. Arena, Kao, C.-C., L. W. Martin and R. Ramesh, Phys. Rev. Lett. 105, 027201 (2010).

[12] H. N. Lee, H. M. Christen, M. F. Chisholm, C. M. Rouleau and D. H. Lowndes, Nature 433, 395 (2005).

[13] G. Sassi, J. Appl. Phys. 54, 5421 (1983); M. Konagai and K. Takahashi, J. Appl. Phys. 46, 3542 (1975).

[14] S. Thiel, G. Hammerl, A. Schmehl, Schneider, C. W. and J. Mannhart, Science 313, 1942 (2006).

[15] A. Brinkman, M. Huijben, M. van Zalk, J. Huijben, U. Zeitler, J. C. Maan, W. G. van der Wiel, G. Rijnders, D. H. A. Blank and H. Hilgenkamp, Nature Mater. 6, 493 (2007).

[16] N. Reyren, S. Thiel, A. D. Caviglia, L. F. Kourkoutis, G. Hammerl, C. Richter, C. W. Schneider, T. Kopp, A.S. Ruetschi, D. Jaccard, M. Gabay, D. A. Muller, J.-M. Triscone and J. Mannhart, Science 317, 1196 (2007)

[17] A. D. Caviglia, S. Gariglio, N. Reyren, D. Jaccard, T. Schneider, M. Gabay, S. Thiel, G. Hammerl, J. Mannhart and J.-M. Triscone, Nature 456, 624 (2008).

[18] C. Cen, S. Thiel, G. Hammerl, C. W. Schneider, K. E. Andersen, C. S. Hellberg, J. Mannhart and Levy, J. Nanoscale control of an interfacial metal-insulator transition at room temperature. Nature Mater. 7, 298 (2008).

[19] N. Nakagawa, H. Y. Hwang and D. A. Muller, Nature Mater. 5, 204-209 (2006).

[20] G. Singh-Bhalla, C. Bell, J. Ravichandran, W. Siemons, Y. Hikita, S. Salahuddin, A. F. Hebard, H. Y. Hwang and R. Ramesh, Nature Phys. 7, 80 (2011).

[21] P. Blaha, K. Schwarz, G. K. H. Madsen, D. Kvasnicka, and J. Luitz, WIEN2k, An Augmented Plane Wave + Local Orbitals Program for Calculating Crystal Properties (Techn. Universität Wien, Vienna, Austria, 2001). ISBN 3-9501031-1-2

[22] J. P. Perdew, A. Ruzsinszky, G. I. Csonka, O. A. Vydrov, G. E. Scuseria, L. A. Constantin, X. Zhou and K. Burke, Phys. Rev. Lett. 100, 136406 (2008)

[23] C. Ambrosch-Draxl and J. Sofo, Comp. Phys. Comm. 
175, 1 (2006).

[24] See attached Supplemental Material for technical details on the DFT calculations including a discussion of excitonic effects, as well as additional results on the gap-graded $\mathrm{LaFeO}_{3}\left|\mathrm{LaVO}_{3}\right| \mathrm{SrTiO}_{3}$ structure and potential gradient in $\mathrm{LaVO}_{3} \mid \mathrm{SrTiO}_{3}$.

[25] M. De Raychaudhury, E. Pavarini and O. K. Andersen, Phys. Rev. Lett. 99, 126402 (2007).

[26] T.-H. Arima, Y. Tokura, and J. B. Torrance, Phys. Rev. B 48, 17006 (1993); T.-H. Arima and Y. Tokura, Journ. Phys. Soc. Japan 64, 2488 (1995). We calculated the absorption coefficient from the reported reflectivity using Kramers-Kronig relations following Ref. 32
[27] H. Weng and K. Terakura, Phys. Rev. B 82, 115105 (2010).

[28] R. Claessen (private communication).

[29] Y. Hotta, T. Susaki and H. Y. Hwang, Phys. Rev. Lett. 99, 236805 (2007).

[30] S. Okamoto, A. J. Millis and N. A. Spaldin, Phys. Rev. Lett. 97, 056802 (2006)

[31] F. Tran and P. Blaha, Phys. Rev. Lett. 102, 226401 (2009).

[32] Data analysis package Datan by C. Porter and D. Tanner, http://www.phys.ufl. edu/ tanner/datan.html 


\title{
Oxide heterostructures for efficient solar cells — Supplementary Information
}

\author{
Elias Assmann, ${ }^{1}$ Peter Blaha, ${ }^{2}$ Robert Laskowski, ${ }^{2}$ Karsten Held, ${ }^{1}$ Satoshi Okamoto, ${ }^{3}$ and Giorgio Sangiovanni ${ }^{4}$ \\ ${ }^{1}$ Institute of Solid State Physics, Vienna University of Technology, 1040 Vienna, Austria \\ ${ }^{2}$ Institute of Materials Chemistry, Vienna University of Technology, 1040 Vienna, Austria \\ ${ }^{3}$ Materials Science and Technology Division, Oak Ridge National Laboratory, Oak Ridge, Tennessee 37831, USA \\ ${ }^{4}$ Institut für Theoretische Physik und Astrophysik, Universität Würzburg, 97074 Würzburg, Germany
}

(Dated: April 9, 2013)

The DFT calculations were performed using the full-potential linearized augmented plane-wave code WIEN2k [21], with the PBEsol exchange-correlation potential $[22]$ and a local Coulomb interaction term $U$ on $\mathrm{Ti}, \mathrm{V}$ and $\mathrm{Fe}\left(U_{\mathrm{Ti}}=9.8 \mathrm{eV}, U_{\mathrm{V}}=3 \mathrm{eV}, U_{\mathrm{Fe}}=5 \mathrm{eV}\right.$, i.e. a GGA+U scheme. The $U$ values have been selected to reproduce the experimental band gaps in the bulk, which is essential in this case since the heterostructures would become conducting too soon (i.e. the critical thickness would be too small) if the gaps were smaller.

This is also the rationale for the large $U_{\mathrm{Ti}}$ : It is necessary to shift the Ti- $d$ states up far enough; but because they are almost empty, this drastic value is needed to achieve the desired effect. This is analogous to the case of $\mathrm{LaTiO}_{3}$, where a large $U$ on the empty La- $f$ states is necessary to shift them away from Ti- $d$.

An independent verification of the results achieved with this $U_{\mathrm{Ti}}$ comes in the form of a calculation using the parameter-free modified Becke-Johnson (mBJ) exchangecorrelation potential [31], which we find to qualitatively agree with the corresponding DFT $+\mathrm{U}$ calculation.

Formally, periodic boundary conditions are in force in all our calculations; however, in the thin-film case the vacuum acts to separate $\mathrm{LaVO}_{3}$ and $\mathrm{SrTiO}_{3}$, effectively imposing open boundary conditions.

The lattice constants of $\mathrm{SrTiO}_{3}, \mathrm{LaVO}_{3}$, and $\mathrm{LaFeO}_{3}$ are quite similar when one takes into account that the unit cells of the latter two are enlarged with respect to the primitive cubic perovskite cell due to the reduced symmetry; they are doubled in the $z$-direction, and form a $\sqrt{2}$-cell in the $x y$-plane. We take this $\sqrt{2}$ setting for the heterostructure unit cell in the $x y$-plane, thus there are two $\mathrm{Ti} / \mathrm{V} / \mathrm{Fe}$ atoms per plane. We fix the in-plane lattice parameter to the value in the $\mathrm{SrTiO}_{3}$ substrate (this corresponds to the experimental situation), while the extent in $z$-direction for the multi-layer structures corresponds to the sum of the bulk layer separations. For the corresponding thin-film structure, this value is enlarged by $20 \mathrm{Bohr} \approx 10.6 \AA$. Within this volume, all atomic positions are relaxed. For the numerical values, see Supplemental Table I.

In the bulk, both $\mathrm{LaVO}_{3}$ and $\mathrm{LaFeO}_{3}$ show antiferromagnetic $(\mathrm{AF})$ spin and orbital order at low temperatures: $\mathrm{LaVO}_{3}$ has AF-C spin and AF-G orbital order while $\mathrm{LaFeO}_{3}$ has AF-G order in both channels; DFT predicts this in accordance with experiment. This raises
Supplemental Table I: Lattice constants of bulk $\mathrm{SrTiO}_{3}$ (cubic), $\mathrm{LaVO}_{3}$ (orthorhombic), and $\mathrm{LaFeO}_{3}$ (orthorhombic). $a$ and $b$ are the in-plane $\sqrt{2}$ lattice parameters, while $c$ is the $\mathrm{BO}_{2}$-plane separation.

\begin{tabular}{lccc}
\hline & $a / \AA$ & $b / \AA$ & $c / \AA$ \\
\hline $\mathrm{SrTiO}_{3}$ & 5.523 & 5.523 & 3.905 \\
$\mathrm{LaVO}_{3}$ & 5.539 & 5.560 & 3.907 \\
$\mathrm{LaFeO}_{3}$ & 5.554 & 5.568 & 3.928 \\
\hline
\end{tabular}

the question which spin order prevails in the heterostructures. For $\mathrm{LaVO}_{3} \mid \mathrm{SrTiO}_{3}$, we focus on AF-C both in analogy to the bulk, and because it has the lowest energy in DFT (among AF-A, AF-C, AF-G and FM) in two test cases.

When a second magnetic material comes into play with $\mathrm{LaFeO}_{3}$, the situation becomes more complicated and three different spin orders have to be considered in general: within $\mathrm{LaVO}_{3}$, within $\mathrm{LaFeO}_{3}$ and at the interface. In the $\mathrm{LaFeO}_{3}\left|\mathrm{LaVO}_{3}\right| \mathrm{SrTiO}_{3}$ calculation presented here, there is only one layer of each material, therefore we need to consider only the coupling between $\mathrm{LaFeO}_{3}$ and $\mathrm{LaVO}_{3}$, which we choose antiferromagnetic in the interest of comparability with the $\mathrm{LaVO}_{3}$-only calculations, resulting in "AF-C" spin order across $\mathrm{V}$ and Fe.

The absorption coefficient for $\mathrm{LaVO}_{3}$ was calculated within GGA $+\mathrm{U}$ as described above, using the program of Ref. 23 while for the semiconductors the mBJ potential was used [31], which yields band gaps in good agreement with the experimental values in these cases. The calculated absorption coefficients have been checked regarding convergence with respect to the density of $k$-points used to sample the Brillouin zone. For the heterostructure, up to $10 \times 10 \times 1 k$-points were used.

The electron-hole interaction, which we investigated by solving the Bethe-Salpeter equation for bulk $\mathrm{LaVO}_{3}$, introduces a sizable $(\sim 0.6 \mathrm{eV})$ but rigid shift of the spectrum to lower energies. However, since the shape of the optical absorption is hardly changed, a somewhat larger value of $U_{\mathrm{V}}$ could easily compensate this shift, reproducing once again the experimental value for the optical gap as in Fig. 5 in the main paper. Thus we conclude that electron-hole interactions do not change our predictions significantly.

The experimental absorption coefficient for $\mathrm{LaVO}_{3}$ was 


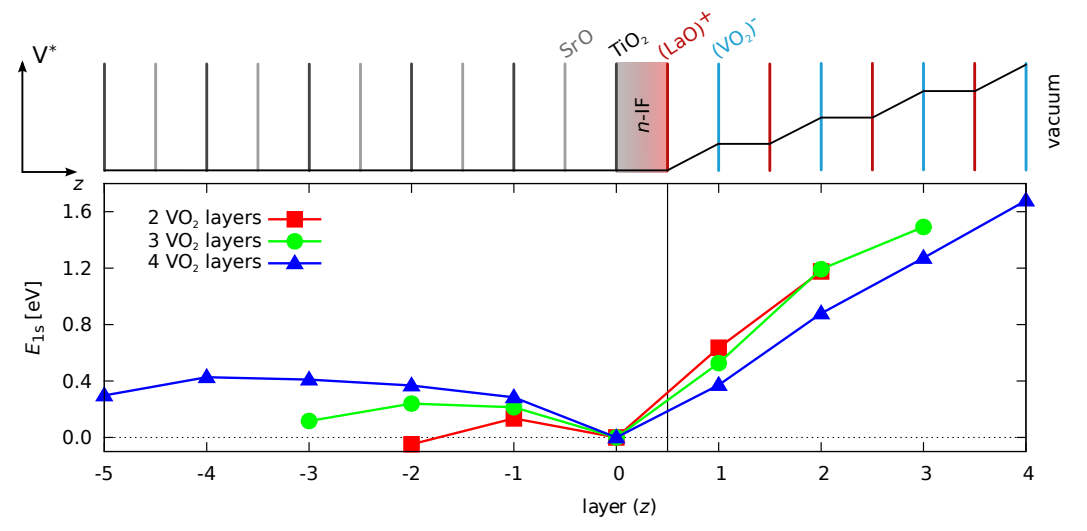

Supplemental Figure 1: Potential gradient in thin-film $\mathrm{LaVO}_{3} \mid \mathrm{SrTiO}_{3}$ heterostructures. The curves $(\mathbf{\square} / \bullet / \Delta$ distinguish structure size) track the energy of an O1s state through the $\mathrm{TiO}_{2}$ (layer $\leq 0$ ) and $\mathrm{VO}_{2}$ (layer $>0$ ) layers, which provides a measure of the potential gradient. Above the data, the layers, interfaces (IF), and effective electron potential $V^{*}$ are shown schematically. The potential in the $\mathrm{SrTiO}_{3}$ part is almost flat, showing only a slight buckling which we attribute to band bending at the interface. calculated from the reflectivity measured in Ref. 26 using Kramers-Kronig relations, employing the datan program package [32].

Gradient in the thin-film case. In analogy to Fig. 4 in the main paper, we show the behavior of the O-1s level in thin-film heterostructures in Supplemental Fig. 1.

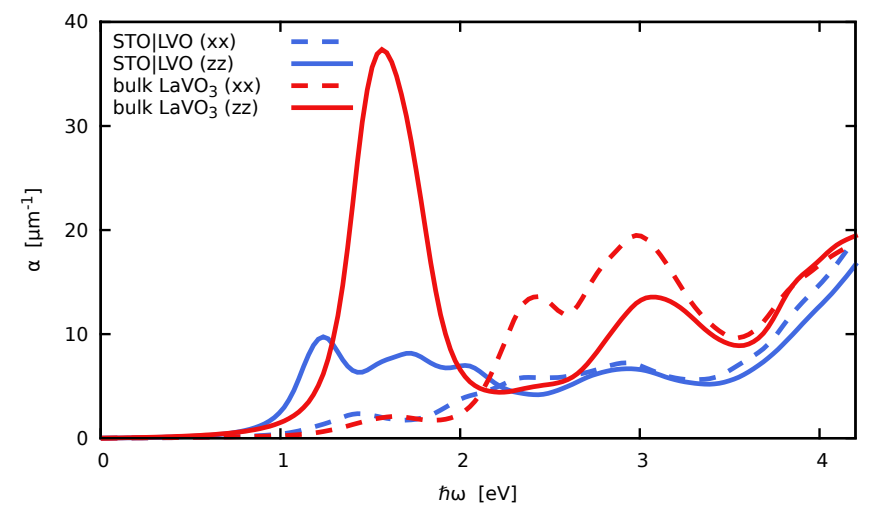

Supplemental Figure 2: Contributions to absorption coefficients. Both in bulk $\mathrm{LaVO}_{3}$ and the heterostructure, a significant asymmetry is seen between the $x x$ and the $z z$ components, while $y y$ is similar to $x x$. The off-diagonal components are rather small in comparison with the diagonal ones.

Absorption coefficients. As stated in the main text, the absorption coefficient $\alpha(\omega)$ is defined via the exponential dampening of the light intensity as a function of distance travelled through the material. However, in addition to the frequency dependency, the absorption also depends on the light's polarization, leading to a matrix $\alpha_{i j}$ analogous to the dielectric tensor. In cubic crystals (such as CdTe and GaAs), symmetry reduces the tensor to only one independent component, $\alpha_{i i}$, and this is what is plotted for these materials in Fig. 5 in the main paper.

On the contrary, in $\mathrm{LaVO}_{3}$ and $\mathrm{LaVO}_{3} \mid \mathrm{SrTiO}_{3}$, the reduced symmetry allows six independent entries. Which of these may contribute to the actual absorption depends of course on the light's angle of incidence with respect to the crystal. Since the heterostructure is grown along the $z$-direction, in the first approximation the po- larization will be in the $x y$ plane. However, because some of the light will come from other directions due to scattering, and because heterostructures are often grown on a stepped substrate, some z-component will also be present, and all $\alpha_{i j}$ may contribute. Therefore, we plotted a suitably averaged quantity $\bar{\alpha}:=\frac{1}{3}\left(\sum_{i} \alpha_{i i}+\sum_{i j} \alpha_{i j}\right)$ in Fig. 5. Supplemental Fig. 2 shows some of the individual contributions. The optical absorption for the heterostructure was calculated in the multi-layer geometry with two $\mathrm{VO}_{2}$ layers.

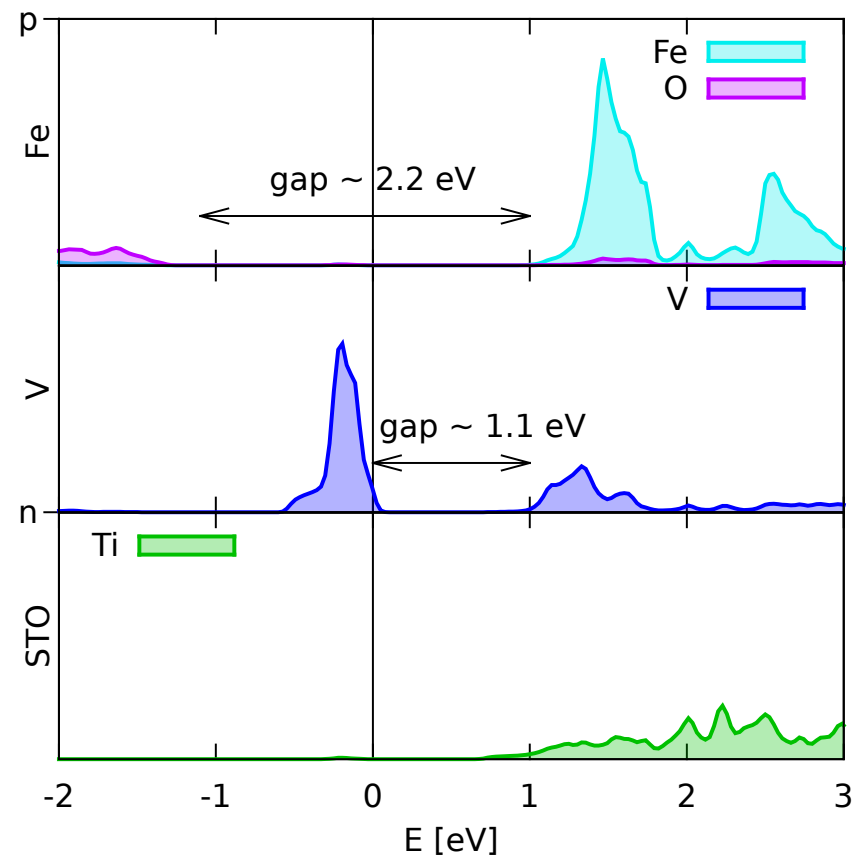

Supplemental Figure 3: The relevant contributions to the density of states around the Fermi level for the "gap-graded" $\mathrm{LaFeO}_{3}\left|\mathrm{LaVO}_{3}\right| \mathrm{SrTiO}_{3}$ structure. In this case, the two V/Fe sites give almost identical contributions (in opposite spin channels). A $p$ - and an $n$-type interface appear, as marked, due to periodic boundary conditions.

Band-gap grading with $\mathbf{L a F e} \mathrm{O}_{3}$. In this section, we give details on the calculation combining $\mathrm{LaVO}_{3}$ and $\mathrm{LaFeO}_{3}$ referenced in the main text. The structure con- 
tains $3 \mathrm{Ti}, 1 \mathrm{~V}$ and $1 \mathrm{Fe}$ layer, with two metal atoms per layer. The projected density of states for Fe, $\mathrm{V}$ and the Ti layer at the $n$-type interface is shown in Supplemental Fig. 3. The differing magnitudes of the $\mathrm{Fe}-\mathrm{O}$ and $\mathrm{V}-\mathrm{V}$ gaps are clearly seen.

It should be emphasized that $\mathrm{LaVO}_{3}+\mathrm{LaFeO}_{3}$ is only one example for many different combinations that may be envisaged within the flexible framework provided by oxide heterostructures.

Acknowledgments. We thank Andrei Pimenov for the calculation of the experimental $\mathrm{LaVO}_{3}$ absorption.

[21] P. Blaha, K. Schwarz, G. K. H. Madsen, D. Kvasnicka, and J. Luitz, WiEN2k, An Augmented Plane Wave + Lo- cal Orbitals Program for Calculating Crystal Properties (Techn. Universität Wien, Vienna, Austria, 2001). ISBN 3-9501031-1-2

[22] J. P. Perdew, A. Ruzsinszky, G. I. Csonka, O. A. Vydrov, G. E. Scuseria, L. A. Constantin, X. Zhou and K. Burke, Phys. Rev. Lett. 100, 136406 (2008)

[23] C. Ambrosch-Draxl and J. Sofo, Comp. Phys. Comm. 175, 1 (2006).

[26] T.-H. Arima and Y. Tokura, Journ. Phys. Soc. Japan 64, 2488 (1995).

[31] F. Tran and P. Blaha, Phys. Rev. Lett. 102, 226401 (2009).

[32] Data analysis package Datan by C. Porter and D. Tanner, http://www.phys.ufl. edu/ tanner/datan.html 